\title{
The effectiveness and appropriateness of Interval Laparoscopic Appendectomy on adult appendiceal mass, a retrospective cohort
}

Shiro Fujihata ( $\nabla$ shiro-1110shiro@hotmail.co.jp)

Nagoya Shiritsu Daigaku https://orcid.org/0000-0001-5410-3724

Hidehiko Kitagami

Kariya Toyota General Hospital

Yosuke Kitayama

Kariya Toyota General Hospital

Ayumi Suzuki

Kariya Toyota General Hospital

Moritsugu Tanaka

Kariya Toyota General Hospital

Shunsuke Hayakawa

Nagoya Shiritsu Daigaku

Seiichi Nakaya

Nagoya Shiritsu Daigaku

Tomotaka Okubo

Nagoya Shiritsu Daigaku

Hiroyuki Sagawa

Nagoya Shiritsu Daigaku

Tatsuya Tanaka

Nagoya Shiritsu Daigaku

Hiroki Takahashi

Nagoya Shiritsu Daigaku

Yoichi Matsuo

Nagoya Shiritsu Daigaku

Ryo Ogawa

Nagoya Shiritsu Daigaku

Shuji Takiguchi

Nagoya Shiritsu Daigaku

Research article 
Keywords: Laparoscopic appendectomy, Interval appendectomy, Laparoscopic interval appendectomy, Appendiceal mass

Posted Date: September 8th, 2019

DOI: https://doi.org/10.21203/rs.2.14168/v1

License: (1) This work is licensed under a Creative Commons Attribution 4.0 International License. Read Full License 


\section{Abstract}

Background: Appendiceal mass sometimes requires extended resection and difficult to treat postoperative complications. Interval appendectomy has been performed mainly in the field of pediatric surgery to avoid them. Recently, there are a few reports on interval laparoscopic appendectomy (ILA). We examined not only the safety and effectiveness of ILA for adult appendiceal mass (AAM), but also the appropriateness of ILA for which cases should be treated non-surgically for ILA or converted to surgical treatment.

Methods: Among 956 appendectomies performed between April 2012 and March 2018, there were 49 cases of AAM, of which 34 cases underwent ILA; ILA group. The safety and efficacy of ILA were examined by comparing with 477 cases of adult uncomplicated appendicitis underwent laparoscopic appendectomy (LA); LA group. The appropriateness of ILA was examined by comparing with the remaining 15 cases of AAM; non-ILA group.

Results: The ILA group had a longer operation time than the LA group, but the postoperative hospital stay was shorter, and no significant difference were found in the bleeding volume, the days required for oral intake of solid food, and the perioperative complications. Examining the factors for failure of non-surgical treatment or relapse of inflammation during interval period, higher WBC day3 / day0 was found to be an independent risk factor.

Conclusion: ILA against AAM was almost as safe and effective as LA for adult uncomplicated appendicitis. WBC day3 / day 0 may be effective as an objective parameter for judging the termination of non-surgical treatment for ILA. This determination to convert ineffective non-surgical treatment to surgical treatment earlier may reduce the burden on the patient.

\section{Background}

Appendicitis is a common disease, but depending on the degree of inflammation, it may be difficult to decide treatment strategies as well as dealing with the postoperative complications. In particular, appendiceal mass (AM) is difficult to treat. AM results from a walled-off appendiceal perforation and represents a wide pathological spectrum ranging from an inflammatory mass that consists of the inflamed appendix, some adjacent viscera and the greater omentum to a periappendiceal abscess [1]. The treatment strategy for AM has long been debated. Typical treatments include the following: 1) early surgery, 2) non-surgical treatment without surgery, and 3) non-surgical treatment followed by interval appendectomy (IA) [2]. In recent years, especially in the field of pediatric surgery, IA has been often selected to reduce the risk of surgical complications $[3,4]$. Furthermore, in the field of surgical techniques, laparoscopic appendectomy (LA) has been widely performed. We have been performing Interval Laparoscopic Appendectomy (ILA) as a first-choice treatment against adult appendiceal mass (AAM), but the safety and efficacy of ILA, as well as the appropriateness of ILA for which cases should be treated 
non-surgically for ILA or converted to surgical treatment when non-surgical treatment is not effective, is unclear.

In this study, we aimed to elucidate the safety, efficacy, and appropriateness of ILA against AAM.

\section{Methods}

Patients who underwent appendectomy at our institute during April 2012 and March 2018 were examined retrospectively. There were 956 cases of appendectomy during the period. Among them, 56 cases were diagnosed as AM, of which 49 were adult cases. Of these, 34 cases were performed ILA, they were defined as the ILA group. Then, 477 cases underwent LA for adult uncomplicated appendicitis were defined as the LA group. And, the remaining 15 cases of AAM were defined as the non-ILA group [Fig. 1]. The ILA group and the LA group was compared to elucidate the safety and efficacy of ILA. From the comparison between the ILA group and the non-ILA group, the risk factors for failure of non-surgical treatment or relapse of inflammation during interval period was examined, and cases in which ILA should be peformed were considered.

General physical conditions are classified according to ASA classification, and medical histories are represented according to Charleston comorbidity index. Clinical variables are represented by median, with 1st and 3rd quartile deviation. Mann-Whitney U-test was used for comparing the clinical variables. Categorical variables are represented by numbers, Fisher's exact test was used for comparing categorical data. Logistic regression analysis was used for univariate and multivariate analysis to examine the variables related to failure of non-surgical treatment or relapse of inflammation during interval period. The variables for multivariate analysis were decided by considering the results of the univariate analysis and of previous reports $[5,6]$. The Cut off value was obtained from the ROC curve, and the point where the sum of sensitivity and specificity was the highest was taken. All statistical analyses were performed with EZR (Saitama Medical Center, Jichi Medical University, Saitama, Japan), and P-value $<0.05$ was judged to be statistically significant.

Therapeutic flowchart of appendicitis

Diagnosis of appendicitis

All patients were asked detailed questions about their symptoms and medical history, followed by palpation of the abdomen and blood tests. Diagnosis of the appendicitis and AM was made by abdominal CT, and a contrast medium was used in many cases except for patients with chronic or acute renal failure. Those with a mass of $30 \mathrm{~mm}$ or more were diagnosed to have AM. The initial imaging diagnosis was conducted by the surgeon and confirmed by a radiologist. In the same day, LA was performed for those with uncomplicated appendicitis (LA group), or with sepsis or non-drainable pelvic abscess among AM (non-ILA group).

Non-surgical treatment for appendiceal mass 
Broad spectrum antibiotics, Piperacillin4g-tazobactam0.5g every 8 hours, were administered primarily through intravenous drip, and drugs were reconsidered according to the results of the microbial susceptibility tests. Drainage of the abscess was considered as soon as possible under CT guidance, and temporal aspiration of the abscess was performed when it was difficult to place the drainage tube. When high fever or abdominal pain persisted, or when the blood test showed an inadequate response to the treatment, the antibiotics were changed and reassessment of the mass was made using CT imaging. Cases that did not show improvement during the non-surgical treatment were treated with surgery (nonILA group). After discharge from the non-surgical treatment, no oral antibiotics were prescribed and a colonoscopy was recommended to exclude malignant diseases. LA was performed 3-4 months after discharge from the initial non-surgical treatment (ILA group). In cases inflammation relapsed during the interval period (non-ILA group), LA was performed immediately.

\section{Laparoscopic appendectomy}

The primary surgeon was selected from surgeons with less than 8 years of experience (postgraduate year $10)$, and the surgery was performed by a left-positioned surgeon and an assistant (scopist), with the guidance of a supervising surgeon. All patients were given general anesthesia. A 10-mm or 5-mm skin incision under the umbilicus was performed, and optical access trocar was used for inserting a 10-mm or $5-\mathrm{mm}$ trocar into the intraperitoneal cavity [7]. Pneumoperitoneum with $10-\mathrm{mmHg} \mathrm{CO}_{2}$ pressure was obtained, and a 5-mm trocar was inserted through the left lower abdomen, where a 0-degree 5-mm optical camera was inserted. Next, a 5-mm or 3-mm trocar was inserted through the suprapubic area. After the trocars entry, the patient was placed in the Trendelenburg position, with a 10-degree angle to the left. When necessary the small intestine was moved to the superior side. The closure of the appendix stump was selected from either a single use of Endoloop (PDS-Endoloop ${ }^{\circledR}$ 2-0; Ethicon, Johnson \& Johnson Medical, Switzerland: EL) at the proximal end of the appendix or Endostapler (ENDOPATH® ATW35; Ethicon, Johnson \& Johnson Medical, Switzerland: ES, or The Endo GIA ${ }^{T M} 30 \mathrm{~mm}$ Reload with Tri-Staple ${ }^{T M}$ AMT; Covidien, Mansfield,MA, USA). The fascial defect on the 10-mm trocar site was closed with absorbable sutures.

\section{Results}

In the ILA group, the median interval from discharge of the initial non-surgical treatment to the surgery was 110 days. Demographic and clinical characteristics of patients in the ILA group and the LA group are shown in Table 1. The ILA group was older, used more anticoagulant or antiplatelet agents, and had higher ASA score and Charlson Comorbidity Index than the LA group [age (years): 56.5 vs $37, P<0.01$; anticoagulant or antiplatelet usage (cases): 5 vs $10, P<0.01$; ASA-classification (1/2/3): 14/12/8 vs 279/185/13, $P<0.01$; Charlson Comorbidity Index (0 / 1 / 2 / 3 / 4 / 5 / 6): 22 / 4 / 5 / 2 / 0 / 1 / 0 vs 414 / 40 / 15 / 5 / 1 / 0 / 2, $P<0.01]$. Considering surgical procedures, in the ILA group, Endloop was statistically used and a 10-mm trocar was tended to be used [appendix stump closure (Endostapler/Endoloop): 13/21 vs 298/179; $P<0.01$, use of 10-mm trocar (use/not use): 28/6 vs 440/37, $P=0.055]$. Though the operation time in the ILA group was longer than that of the LA group, the 
postoperative hospital stay was shorter, and there was no difference between the bleeding volume and the nunber of days required until oral intake of solid food after surgery between the two groups [operation time (mins): 64 vs 56, $P<0.01$; postoperative hospital stay (days): 2 vs $3, P<0.01$ ] [Table 2]. Regarding perioperative complications, there was 1 case of small bowel obstruction in the ILA group. In the LA group, there were 12 cases of wound infection, 1 case of anorexia, 1 case of damage to other internal organ at the time of the first trocar insertion, 1 case of transient liver injury. There were no significant differences in the perioperative complications between the two groups.

The median number of days from start of non-surgical treatment to perform the surgery in the non-ILA because of failure of non-surgical treatment or relapse of inflammation was 9 days. The characteristics of the ILA group and the non-ILA group are shown in Table 3, among which WBC day3 / day0 and CRP day3 / day0 showed a statistical difference between the two groups [WBC day3 / day0: 0.67 vs $0.97, P<$ 0.01 ; CRP day3 / day0: 0.58 vs $1.21, P=0.011]$. The univariate analysis indicated mass size and WBC day3 / day0 were considered as factors that could influence the failure of non-surgical treatment or relapse of inflammation during interval period [mass size: $P=0.017$; WBC day3 / day $0: P=0.013$ ] [Table 4]. Furthermore, multivariate analysis considering the results of the univariate analysis indicated that WBC day3 / day 0 is an independent factor that could influence ita [ $P=0.045$ ] [Table 4]. In addition, WBC day3 / day 0 showed the highest sensitivity and specificity at a cut off value of 0.906 [sensitivity 0.750 , specificity 0.794] [Fig. 2]. Using the cut off value, the patients were divided into two groups as the high and low WBC day3 / day0, resulting in a statistically higher proportion of the high WBC day3 / day0 in the non-ILA group than the ILA group $[P<0.01]$ [Table 5].

In comparison between the ILA group and the non-ILA group, significant differences were found in operation time, bleeding volume, postoperative hospital stay, and days required until oral intake of solid food after surgery [operation time (mins): 64 vs 161, $P<0.01$; bleeding volume (ml): 2 vs 200, $P<0.01$; postoperative hospital stay (days): 2 vs $9, P<0.01$; days required until oral intake of solid food (days): 1 vs $3, P<0.01]$. Regarding perioperative complications, there were significant differences between the two groups in conversion to laparotomy, postoperative drainage tube placement, small bowel obstruction

, and postoperative residual abscess [conversion to laparotomy: 0 vs $3, P=0.025$; postoperative drainage tube placement: 0 vs 3, $P=0.025$; small bowel obstruction: 1 vs $4, P=0.026$; residual abscess: 0 vs $4, P<$ 0.01) [Table 6].

Preoperative colonoscopy was performed for 27 of 34 cases in the ILA group, but no neoplastic lesion was found. Postoperative pathological examination revealed 1 case of appendiceal malignant disease in each of the ILA group and the non-ILA group.

\section{Discussion}

Considering that $2-6 \%$ of acute appendicitis are reported to be accompanied by an AM, it is not uncommon to have an opportunity to treat AM [8]. However, no unified view has been obtained so far regarding the treatment strategy for AM. The history of non-surgical treatment and IA for AM is long [9, 
10]. However, since the mainstream of treatment was early appendectomy, there were cases requiring extended resection in approximately 3 to $30 \%$, and the subsequent perioperative complications were considerable problems $[8,11-13]$. In recent years, with the advance of interventional radiology as well as the improvement of preoperative diagnosis, non-surgical treatment for AM has developed dramatically $[14,15]$. Additionally, many reports have referred to the effectiveness of $I A$, especially in pediatric surgery $[3,4,16]$. In non-surgical treatment without appendectomy, there is a risk of relapsing inflammation and potential presence of a malignant disease $[17,18]$. The relapse rate in previous reports are not consistent $[13,19,20]$. Therefore, the necessity of IA cannot be considered by the relapse rate alone. However, the rate of neoplasm has been reported to be 10 to $29 \%$ in patients presenting appendiceal inflammatory mass, it should not be ignored [21]. Furthermore, in this study, many postoperative pathological results of ILA-treated cases exhibited pathological abnormality with chronic inflammation, that is the point Rosen suggested that IA should be performed after non-surgical treatment [22]. The risk of relapse along with the risk of malignant disease, we believe, favors IA after non-surgical treatment for AM.

Considering surgical procedures, LA for uncomplicated appendicitis has been widely performed as a safe operation. LA can be adapted even for complicated appendicitis and the elderly with the standardization of surgery and advancement of techniques [23, 24]. However, the rate of ILA for AM still low [21]. Therefore, there are not many reports on the effectiveness of ILA, and its verification is significant. The present study showed a longer operation time in the ILA group compared with the LA group. However, other intraoperative related variables such as the bleeding volume did not increase, and there was no need for conversion to laparotomy. Cases with non-surgical treatment and interval period usually have disintegrated layers of the appendix as well as the surrounding structures, with $8 \%$ of IA cases being reported to have a melted appendix [18]. In addition, some adhesions remain around the appendix and the abdominal wall even after inflammation improved which makes it difficult to perform surgery as well as identify the appendix [25]. However, even considering the disintegration and the adhesions, the interval allowed the inflammation to improve, enabling us to perform ILA with equal safety as LA for uncomplicated appendicitis, which leads to easier postoperative management of AM as showed in postoperative hospital stay or postoperative complications. Thus, the ILA procedures for AAM was almost as effective as the LA for adult uncomplicated appendicitis.

By comparing the ILA group and the non-ILA group, it was clear that the ILA procedures were less invasive and easier postoperative management than the non-ILA group. When surgery is performed without nonsurgical treatment for AM, surgical complications increase due to the edema, vulnerability of the adjacent small and large intestine, as well as difficulty in approaching the appendix due to deformation of the anatomic structures and location [26]. In order to avoid extended resection and perioperative complications, non-surgical treatment should be completely fulfilled. One tactic to fulfill the ILA procedure is drainage of the abscess. However, among the 9 cases of AAM underwent CT guided drainage in the present study, 2 cases failed to reach ILA (22.2\%). In a previous report, Horn reported that 561 patients (25.4\%) among 2209 cases of AM failed to improve by drainage and required surgical treatments [27]. Whether abscess drainage can be performed or not is not an absolutely critical factor in completing the ILA procedures. Our present study showed that WBC day3 / day 0 value could be a simple clinical 
parameter to estimate the failure of non-surgical treatment or relapse of inflammation during interval period. When the WBC day3 / day0 value during non-surgical treatment is higher than 0.907 , it means that the residual non-surgical treatment may not be effective. As our study is a small retrospective study, the value of 0.907 will still need a further assessment by a larger prospective study, preferably randomized. Nevertheless, a clinical index of WBC day3 / day0 has a potential of an effective parameter to avoid the burden on patients and extension of hospital stay caused by continuing ineffective treatment.

\section{Conclusions}

This examination showed that ILA against AAM was almost as safe and effective as LA for adult uncomplicated appendicitis. However, not all patients can be treated with ILA as a treatment for AAM. WBC day3 / day 0 is an independent parameter for predicting the failure of non-surgical treatment or relapse of inflammation during interval period and terminating ineffective non-surgical treatment for ILA.

\section{Declarations}

\section{Availability of data and materials}

The datasets used and/or analysed during the current study are available from the corresponding author on reasonable request.

\section{Abbreviations}

ILA Interval laparoscopic appendectomy

AAM Adult appendiceal mass

LA Laparoscopic appendectomy

AM Appendiceal mass

\section{Acknowledgements}

Not applicable.

\section{Authors' Contributions}

Study conception and design: SF, TT, RO, HT, YM, and HK. Acquisition of data: SF, YK. Analysis and interpretation of data: SF, SN, SH, TO, and HS. Drafting of manuscript: SF, AS, and HK. Critical revision of manuscript: HK and ST. All authors read and approved the final manuscript. 


\section{Funding}

The present study received no fund support.

\section{Ethics approval and consent to participate}

Our investigation was carried out according to the Declaration of Helsinki. This retrospective study was approved by Kariya Toyota General Hospital institutional review board (No.518). Informed consent was obtained from all individual participants included in the study.

\section{Consent for publication}

Not applicable.

\section{Competing interests}

The authors declare that they have no competing interests.

\section{References}

1. Nitecki S, Assalia A, Schein M. Contemporary management of the appendiceal mass. Br J Surg. 1993; 80: 18-20.

2. Friedell ML, Perez-Izquierdo M. Is there a role for interval appendectomy in the management of acute appendicitis? Am Surg. 2000; 66: 1158-62.

3. Hall NJ, Eaton S, Stanton MP, Pierro A, Burge DM. Active observation versus interval appendicectomy after successful non-operative treatment of an appendix mass in children (CHINA study): an open-label, randomized controlled trial. Lancet Gastroenterol Hepatol. 2017; 2: 253-260.

4. Hall NJ, Jones CE, Eaton S, Stanton MP, Burge DM. Is interval appendectomy justified after successful nonoperative treatment of an appendix mass in children? a systematic review. J Pediatr Surg. 2011; 46: 767771.

5. Chang YJ, Chao HC, Chen CL, Chen SY, Yan DC, Tsai MH. C-reactive Protein may Predict the Recurrence of Appendicitis in Children Formerly with Appendiceal Mass after Successful Non-operative Treatment. 
Pediatr Neonatol. 2017; 58: 350-354

6. Marin D, Ho LM, Barnhart H, Neville AM, White RR, Paulson EK. Percutaneous abscess drainage in patients with perforated acute appendicitis: effectiveness, safety, and prediction of outcome. AJR Am J Roentgenol. 2010; 194: 422-9.

7. String A, Berber E, Foroutani A, Macho JR, Pearl JM, Siperstein AE. Use of the optical access trocar for safe and rapid entry in various laparoscopic procedures. Surg Endosc. 2001; 15: 570-3

8. Wright GP, Mater ME, Carroll JT, Choy JS, Chung MH. Is there truly an oncologic indication for interval appendectomy? Am J Surg. 2015; 209: 442-6.

9. JB Murphy. Thousand operations for appendicitis. Am J Med Sci. 1904; 128: 187-211.

10. Soresi AL. TECHNIC OF APPENDECTOMY: DESCRIPTION OF A RATIONAL, SAFE AND EASY TECHNIC OF THE OPERATION FOR ACUTE AND INTERVAL APPENDICITIS. Ann Surg. 1920; 71: 315-46.

11. McPherson AG, Kinmonth JB. Acute appendicitis and the appendix mass. Br J Surg. 1945; 32: 365-70.

12. Tingstedt B, Bexe-Lindskog E, Ekelund M, Andersson R. Management of appendiceal masses. Eur J Surg. 2002; 168: 579-582.

13. Corfield L. Interval appendicectomy after appendiceal mass or abscess in adults: what is "best practice"? Surg Today. 2007; 37: 1-4.

14. Pereira JK, Chait PG, Miller SF. Deep pelvic abscesses in children: transrectal drainage under radiologic guidance. 1996; 198: 393-6.

15. Forsyth J, Lasithiotakis K, Peter M. The evolving management of the appendix mass in the era of laparoscopy and interventional radiology. 2017; 15: 109-115.

16. Vane DW, Fernandez N. Role of interval appendectomy in the management of complicated appendicitis in children. World J Surg. 2006; 30: 51-4.

17. Hoffman J, Lindhard A, Jensen U. Appendix mass: Conservative management without interval appendectomy. Am J Surg. 1984; 148: 379-82.

18. Eriksson S, Granstrom L. Randomized controlled trial of appendectomy versus antibiotic therapy for acute appendicitis. Br J Surg. 1995; 82: 166-9.

19. Dixon MR, Haukoos JS, Park IU, Oliak D, Kumar RR, Arnell TD, et al. An assessment of the severity of recurrent appendicitis. Am J Surg. 2003; 186: 718-22.

20. Wilson-Storey D, Scobic WG. Appendix mass. A 15-year review. Pediatr Surg Int. 1989; 4: 168-70.

21. Teixeira FJR Jr, Couto Netto SDD, Akaishi EH, Utiyama EM, Menegozzo CAM, Rocha MC. Acute appendicitis, inflammatory appendiceal mass and the risk of a hidden malignant tumor: a systematic review of the literature. World J Emerg Surg. 2017; 9; 12: 12

22. Rosen M, Chalupka A, Butler K, Gupta A, Odom SR. Pathologic findings suggest long-term abnormality after conservative management of complex acute appendicitis. Am Surg. 2015; 81: 297-9.

23. Thomson JE, Kruger D, Jann-Kruger C, Kiss A, Omoshoro-Jones JA, Luvhengo T, et al. Laparoscopic versus open surgery for complicated appendicitis: A randomized controlled trial to prove safety. Surg Endosc. 2015; 29: 2027-32.

Page $10 / 13$ 
24. Ferrarese AG, Martino V, Enrico S, Falcone A, Catalano S, Pozzi G, et al. Laparoscopic appendectomy in the elderly: our experience. BMC Surg. 2013;13. doi: 10.1186/1471-2482-13-S2-S22.

25. Verwaal VJ, Wobbes T, Goris RJ. Is there still a place for interval appendectomy? Dig Surg. 1993; 10: 285-8.

26. Demetrashvili Z, Kenchadze G, Pipia I, Ekaladze E, Kamkamidze G. Management of Appendiceal Mass and Abscess. An 11-Year Experience. Int Surg. 2015; 100: 1021-5.

27. Horn CB, Coleoglou Centeno AA, Guerra JJ, Mazuski JE, Bochicchio GV, Turnbull IR. Drain Failure in IntraAbdominal Abscesses Associated with Appendicitis. Surg Infect (Larchmt). 2018; 19: 321-325.

\section{Tables}

Due to technical limitations tables are only available as a download in the supplemental section

\section{Figures}

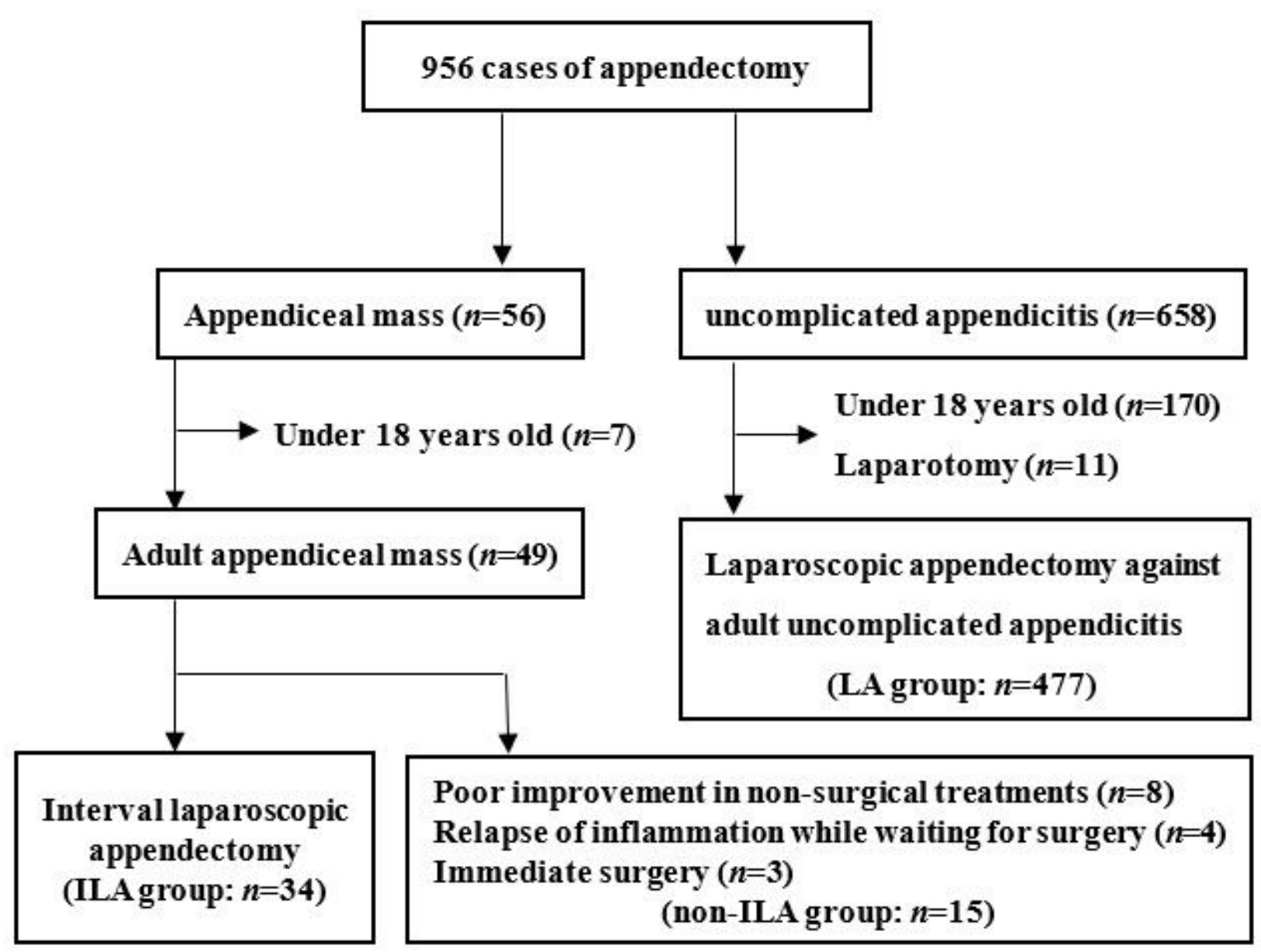


Figure 1

Search strategy

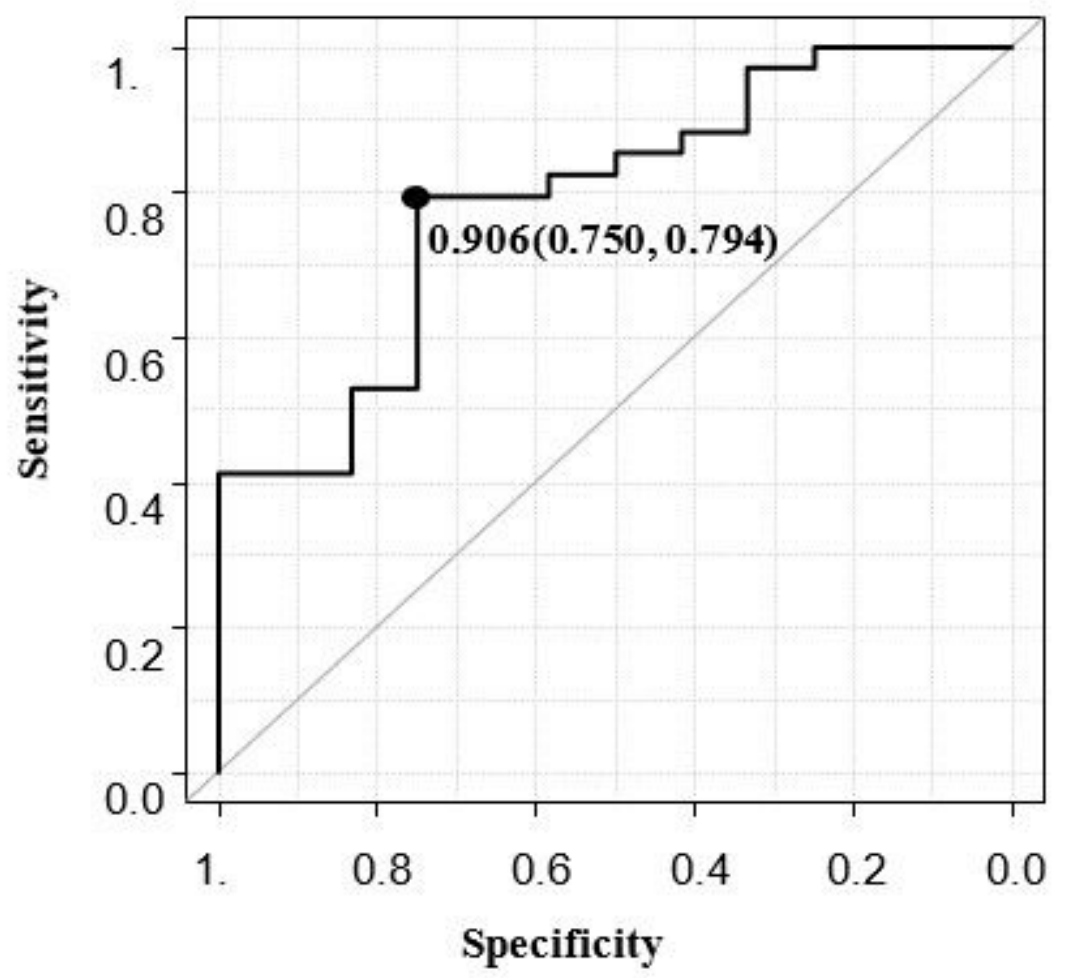

Figure 2

Among adult patients with appendiceal mass, a cutoff value of 0.906 with the highest sensitivity and specificity related with the failure of non-surgical treatment or relapse of inflammation during interval period was determined from ROC curve of WBC day3 / day0.

\section{Supplementary Files}

This is a list of supplementary files associated with this preprint. Click to download.

- TABLE6.docx

- TABLE3.docx

- TABLE1.docx 
- TABLE2.docx

- TABLE5.docx

- TABLE4.docx 